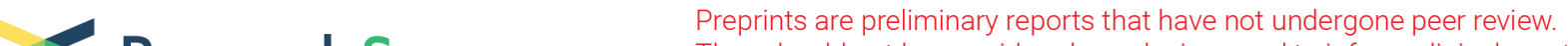 They should not be considered conclusive, used to inform clinical practice, or referenced by the media as validated information.
}

\section{Dissolving Microneedle Rollers for Rapid Transdermal Drug Delivery}

\section{Xiao Peng Zhang}

Beijing University of Chemical Technology

\section{Bao Li Zhang}

Beijing University of Chemical Technology

\section{Bo Zhi Chen}

Beijing University of Chemical Technology

\section{Ze Qiang Zhao}

Beijing University of Chemical Technology

Xin Dong Guo ( $\nabla$ xdguo@buct.edu.cn )

Beijing University of Chemical Technology https://orcid.org/0000-0001-5581-4253

\section{Research Article}

Keywords: Dissolving microneedle roller, diabetic rat, insulin, transdermal drug delivery

Posted Date: April 13th, 2021

DOI: https://doi.org/10.21203/rs.3.rs-413655/v1

License: (c) (i) This work is licensed under a Creative Commons Attribution 4.0 International License. Read Full License

Version of Record: A version of this preprint was published at Drug Delivery and Translational Research on November 6th, 2021. See the published version at https://doi.org/10.1007/s13346-021-01048-8. 


\section{Abstract}

Dissolving microneedle patch (DMNP) is a minimally invasive and painless self-administration device. However, due to "bed-of-needles" effect and skin deformation, it is difficult to apply it on the large areas of skin or curved skin as the patch size increased for DMNP. Here, we propose a polyvinyl alcohol (PVA)based dissolving microneedle roller (DMNR) device that can be used for delivering drugs rapidly on the large surface areas or curved skin and does not need to be attached on the skin all the time during drug delivery. The hypoglycemic effect of insulin-loaded DMNRs for transdermal delivery of insulin was studied on the type 1 diabetic rat models. It was found that the insulin-loaded DMNR has an immediate and effective hypoglycemic effect that the blood glucose level reduced below to $50 \%$ of original blood glucose at 1 hour after DMNRs administrated.

\section{Introduction}

Transdermal drug delivery offers a noninvasive and good patient compliance route compared with subcutaneous (SC) injection, and avoids some other issues via oral route, such as significant first-pass effect of the liver, drug degradation within the gastrointestinal tract (1-3). However, for some biomacromolecule drugs, such as insulin, DNA or vaccine, cannot be delivered into skin effectively by this conventional route because of the strong barrier function of the outermost stratum corneum of skin (4-6). To overcome the stratum corneum limitation, microneedle (MN), one of minimally invasive and painless self-administration drug delivery devices, was introduced to transdermal drug delivery (7). MN is a type of micron-scale needle with diameter in the size of microns and height up to $1000 \mu \mathrm{m}$, which is designed to increase the permeability of drugs to subcutaneous layer of skin by piercing stratum corneum and creating recoverable microchannel on the skin but without stimulating nerves (8-10).

A variety of MNs have been reported in recent years and they can be roughly categorized into five groups (11) including solid MNs (12), coated MNs (13), dissolving MNs (14), swellable MNs (15), and hollow MNs (16). Among these types, dissolving MNs (DMNs) have gained widely attention to deliver protein drugs (17) or for cosmetic applications (18) because DMNs are mainly prepared by some biodegradable water-soluble polymer materials so that the MNs can be quickly and completely dissolved in the skin after contacting with the skin interstitial fluid. Currently, several dissolvable materials are used to fabricate DMNs, such as polyvinylpyrrolidone (PVP) (19), sodium hyaluronate (SH) (20), carboxymethyl cellulose (CMC) (21), and polyvinyl alcohol (PVA) (22). DMNs are generally arranged on a small patch with a thickness to forming a device called dissolving microneedle patch (DMNP) $(23,24)$. However, if the size of DMNP is too large, the DMNP will be difficult to deliver drugs rapidly and efficiently on a large area of skin due to "bed-of-needles" effect (25) and skin deformation (26), especially for the curved skin (27).

Alternatively, microneedle roller (MNR) offers a drug delivery approach over a large area of skin or curved skin surface because of their special geometry $(28,29)$. In this scheme, MNs are assembled on the outer circumferential surface of a cylinder roller and fabricated by metallic or solid materials (30). Generally, 
MNRs are usually used to induce collagen production for cosmetic purposes $(31,32)$ or for drug delivery systems by creating microchannels to increase skin permeability $(33,34)$.

In this work, combining the advantages of DMNs and MNRs, we proposed a novel strategy to fabricate tip drug-loaded dissolving microneedle rollers (DMNRs), which can be used to deliver drugs rapidly and efficiently on large areas of skin or curved skin. PVA, a FDA approved water-soluble polymer, was chosen to fabricate DMNRs instead of metallic or solid materials. The preparation method of DMNR is simple and low-cost. Additionally, this study further evaluated the mechanical properties and rapid drug delivery performances of DMNRs. In vivo studies were performed to test the insertion rates and drug delivery properties of DMNRs that contain different numbers of MNs. To confirm the administration effect, insulinloaded DMNRs were used for diabetic rats and the results suggested that the insulin-loaded DMNRs have good potential to hypoglycemic effect to diabetes.

\section{Materials And Methods}

\section{Materials}

Polyvinyl alcohol (PVA, $M_{W}=9000-10000,80 \%$ hydrolyzed), D (+)-Sucrose $\left(M_{W}=342.29\right.$, purity $\left.\geq 99.9 \%\right)$, Sulforhodamine $B\left(S R B, M_{W}=580.65\right)$, and Citric acid buffer $(0.1 \mathrm{M}, \mathrm{pH}=4.5)$ were purchased from Sigma-Aldrich. Streptozotocin (STZ) was obtained from AbMole Bioscience. Insulin (28 IU/mg) extracted from pig pancreas was purchased from Xuzhou Wanbang Biochemical Pharmaceutical Co., Ltd. Polydimethylsiloxane (PDMS, Sylgard 184), consisting of Base Part A and Curing Agent Part B was purchased from Dow Corning. Other chemicals used were analytical grade without further purification. Porcine cadaver skin was purchased from a local slaughterhouse (Beijing, China).

\section{Methods}

\section{Fabrication of PDMS molds}

PDMS molds with uniform cavities and different dimensions were initially fabricated using a laser-based and micromolding techniques as our previous study proposed (38). PDMS prepolymer solution and curing agent were first mixed in a mold at a ratio of 10:1 (v/v), then carried it out for 30 min under vacuum to remove air bubbles generated by stirring, next cured at $60^{\circ} \mathrm{C}$ for $5 \mathrm{~h}$ to form PDMS sheet. Subsequently, a laser engraving machine (VLS3.50, 50 W, Universal Laser System, USA) was used to prepare a series of conical cavities on the PDMS sheets to form PDMS molds.

\section{Preparation of PVA solution}

PVA powder was firstly dissolved in deionized (DI) water and heated with a magnetic stirring at $90{ }^{\circ} \mathrm{C}$ for $6 \mathrm{~h}$ to obtain $32 \%(\mathrm{w} / \mathrm{w})$ homogeneous solution. Following sucrose (acts as a stabilizer) was added into the solution (sucrose to PVA weight is $3: 4$ ) and kept stirring the mixture at $90^{\circ} \mathrm{C}$ for $1 \mathrm{~h}$ and then cooled it down to room temperature to form $25.8 \%$ PVA mixed solution. 


\section{Fabrication and characterization of DMNRs}

DMNRs were fabricated based on a casting process. Briefly, $200 \mu \mathrm{l}$ of prepared drug solution (insulin solution or $2 \mathrm{mg} / \mathrm{ml} \mathrm{SRB}$ ) was first applied to the PDMS mold under vacuum for 15 minutes. Then, the residual drug solution was removed from the mold surface and the vacuum was continued to concentrate the drugs on the tips of the cavities of PDMS mold. Third, 25.8\% PVA/sucrose solution (about $200 \mathrm{mg}$ ) was cast into the PDMS mold under vacuum for $30 \mathrm{~min}$ at room temperature to make sure PVA/sucrose solution filled with the MN cavities. After that, the mold was dried at room temperature in a fume hood. Subsequently, $3 \mathrm{M}$ double-sided tape with a thickness of $0.2 \mathrm{~mm}$ was attached to the outer circumferential surface of a hollow cylindrical plastic roller with a length of $5 \mathrm{~cm}$, an inner diameter of $2 \mathrm{~mm}$, and an outer diameter of $4 \mathrm{~mm}$. DMNRs were finally formed by demolding the drug-loaded MNs from the PDMS mold using the prepared roller with double-side tape. SRB was used as a model drug, and insulin solution was used as a therapeutic drug for diabetes. Detailed characteristics of DMNRs were observed using fluorescence microscope (SZX7, Olympus, Japan) and scanning electron microscope (SEM, Hitachi S4000, Japan).

\section{Demolding time study of DMNR}

To ensure that the DMNs can be demolded from the PDMS mold without causing any MNs damage, the demolding time of DMNR was first optimized. Briefly, DMNs were demold from PDMS mold after drying 2, 4 , and 8 hours. Each DMNR demolded at different times was then weighed immediately. The relative water content of DMNR demolded at different times was determined by comparing the weight change after immediate demolding and after drying under vacuum for 48 hours.

\section{Mechanical property tests of DMNRs}

Mechanical properties of DMNRs were determined by a displacement-force test station with a mechanical sensor (Mark-10, Force Gauge Model, USA). Both axial force and shear forces of SRB-loaded DMNs with different heights $(400,600$, and $700 \mu \mathrm{m})$ that mounted on the rollers were tested. Briefly, on the one hand, to access the axial compression performance, a DMNR with only one row containing five DMNs was attached to a flat stainless-steel baseplate of the sensor probe while the tips of MNs pointing downwards, and the sensor probe was then lowered in the vertical direction at a speed of $0.1 \mathrm{~mm} / \mathrm{s}$. The data of displacement and force measurements were recorded when the tips of the MNs first touched the smooth stainless-steel platform and continued until the force reached $5 \mathrm{~N}$. On the other hand, to test the shear force of DMNRs, the roller with a single MN was fixed on a vertically placed stainless steel plate, and the tips of MNs faced horizontal direction. The sensor probe was lowered in the vertical direction at a speed of $0.1 \mathrm{~mm} / \mathrm{s}$ while a shear force was then given at $250 \mu \mathrm{m}$ from the tips of the DMNs. Displacement and force measurements were recorded when the sensor probe was first touched the MNs and continued until the shear force was zero. Subsequently, the ultimate shear force was obtained when the MN was fractured under shear force. These experiments were repeated five times to determine the mechanical property of DMNR. After each test, the sensor probe was moved upwards at a speed of $10 \mathrm{~mm} / \mathrm{min}$, and DMNs were then visualized using fluorescence microscope. 


\section{Insertion studies of DMNRs on porcine cadaver skin}

To assess the in vitro insertion abilities of DMNRs with DMN heights of 400,600 , and $700 \mu \mathrm{m}$, porcine cadaver skin without subcutaneous tissues was used as a human skin model. Before experiments, skin was separated into small pieces of $5 \times 5 \mathrm{~cm}$ and stored in a refrigerator at $-20{ }^{\circ} \mathrm{C}$. Prior to insertion test, the skin was first immersed in PBS and thawed for 30 minutes, and excess moisture on the skin surface was absorbed using commercial tissue papers, and then the hair of the pig skin was carefully removed by a disposable razor. SRB-loaded DMNRs were installed on a homemade applicator and then rolled forward once row by row across the skin. To control the force applied on the DMNRs, we referred to the method of previous study (30) that placed the skin on a balance which DMNRs were applied. The skin surface was imaged by fluorescence microscope after DMNR insertion. To clearly evaluate the insertion depth of DMNRs with different height DMNs, the skin samples were sliced from the middle of the DMNs insertion position points and viewed from the side using fluorescence microscope.

\section{Insertion studies of DMNRs on Parafilm M®}

To further observe the holes created of DMNR visually, Parafilm $\mathrm{M}^{\circledR}$, a validated skin model (39), was used as an artificial skin simulants. The Parafilm $\mathrm{M}^{\circledR}$ sheet was folded to get an eight-layer film (appromiximately $127 \mu \mathrm{m}$ thickness per layer). Following, SRB-loaded DMNR (375 DMNs, $600 \mu \mathrm{m}$ ) was installed on a homemade applicator and rolled forward once across the eight-layer film row by row. After insertion, the sheet was unfolded and observed using a handheld microscope (AD7013MZT, Dino-lite, Taiwan, China). The number of holes created in each layer by DMNRs were then calculated under microscope after washing off the fractured MNs in the sheet.

\section{Rapid drug delivery performance tests of DMNRs}

The in vitro drug delivery performance of DMNRs were investigated by controlling the insertion time and insertion ways. Porcine cadaver skin described above was used as a skin model. For easy observation, DMNPs and DMNRs with only one row ( $5 \mathrm{MNs} / \mathrm{row}, 600 \mu \mathrm{m}$ height) were used to insert the skin, respectively. The experiments were divided into two groups: (1) DMNP with $5 \mathrm{MNs} /$ row was vertically inserted into the skin and then removed vertically at 1 second; (2) DMNR was inserted into the skin by rolling it once across the skin and removed within 1 second. Immediately, top views and side views of the skin after insertion were imaged using fluorescence microscope. DMNs were visualized using fluorescence microscope and SEM before and after insertion. The DMNs for each group had the same geometry. Each group repeated five times. Moreover, the model drug does not diffuse immediately in a short time after being transported into the skin by DMNs, so the insertion situation can be evaluated by observing the number of red spots left after the drug was delivered into the skin.

\section{Insertion rate and drug delivery efficiency tests of DMNRs in vivo}

To assess and compare the in vivo insertion and drug delivery performances of DMNRs and traditional DMNPs, the insertion rate and drug delivery efficiency were measured. UV-sterilized DMNRs contains 
different number of SRB-loaded DMNs $(225,375,525)$ were separately applied to the dorsal skin (with a certain curvature) of female SD rats, and the DMNPs with the same geometry and same number of DMNs to DMNRs were fabricated as control group. Prior to the experiment, the dorsal region hair of rats was first removed using electric clippers and depilatory cream under anesthesia, and then cleaned by $75 \%$ ethanol. Notably, DMNRs were rolled once across the dorsal skin and removed immediately, while DMNPs were inserted into the skin and removed after 2 minutes. Subsequently, the skin of insertion sites was immediately observed by handheld microscope, and the amounts of red spots created by SRB-loaded DMNRs or DMNPs on the insertion sites were counted to calculate the insertion rate by the following equation:

\section{Insertion rate $=\frac{\text { the number of red spots on the skin after insertion }}{\text { the number of microneedles before insertion }}$}

At the same time, collecting the drug residues remained on the rollers and patches respectively, as well as residual drug on the insertion sites administrated by DMNRs and DMNPs, to measure the fluorescence intensity using a fluorescence microplate reader (Fluoroskan Ascent 374, Thermo Scientific), and then the amounts of residual drug were calculated. Drug loading of per DMNR or DMNP was also determined using fluorescence microplate reader Therefore, drug delivery efficacy was calculated according to the following equation:

Drug delivery efficiency $=\frac{\text { drug loading-drug residual }}{\text { drug loading }}$

\section{In vivo visualization of drug delivery of DMNR using IVIS imaging tool}

To visualize cutaneous permeation of model drug in live SD rats, the live anesthetized rats were first treated with the same size model drug-loaded DMNRs and DMNPs (600 $\mu \mathrm{m}$ height), respectively. Then imaging of the insertion sites of the rat skin at different times $(0,2,4,6$, and $8 \mathrm{~h})$ after treatment of DMNRs or DMNPs using a non-invasive analytical in vivo imaging system (IVIS, Xenogen 200, Caliper Life Sciences, Hopkinton, MA) to visualize cutaneous permeation of drug in live rats.

\section{Rat model of Type I diabetes}

Sprague-Dawley (SD) rats (6-8 weeks, 200-230 g) were purchased from Beijing Vital River Laboratory Animal Technology Co., Ltd. Rats were housed in laminar flow cages, provided with ad libitum access to food and water, and maintained in a specific environment with temperature of $22 \pm 1{ }^{\circ} \mathrm{C}$, relative humidity of $50 \pm 10 \%$, and a $12 \mathrm{~h}$ light-dark cycle. All rats were allowed to acclimatize the laboratory environment for 1 week before experiments. After overnight fasting, Type 1 diabetic models were induced in female SD rats by single intraperitoneal injection with $75 \mathrm{mg} / \mathrm{kg}$ streptozotocin in $200 \mu$ l sodium citrate buffer (0.1 $\mathrm{M}, \mathrm{pH}$ 4.5). Rats with fasting blood glucose levels exceeding $300 \mathrm{mg} / \mathrm{dl}$ were confirmed as Type 1 diabetic rats. All procedures of animal studies were conducted in accordance with the guidelines of China-Japan Friendship Hospital for care and use of laboratory. 


\section{Blood glucose control studies in type 1 diabetic rats}

Prior to the experiment, type 1 diabetic rats were fasted for $6 \mathrm{~h}$ but received water ad libitum, and the back hair of each group was shaved using an electric shaver under anesthesia. Then all animals were casually divided into the following 3 groups ( $n=4$ for each group): (1) control group: DMNRs without insulin loaded were applied to the dorsal skin of diabetic rats; (2) subcutaneous (SC) group: diabetic rats were injected $0.3 \mathrm{IU}$ insulin solution subcutaneously by disposable insulin syringes on the dorsal skin of diabetic rats; (3) DMNRs group: insulin-loaded DMNRs ( $0.3 \mathrm{IU}$ per roller) were applied to the dorsal skin of diabetic rats. The blood glucose levels of rats in each group were measured using One Touch glucometer before and at $0.5,1,1.5,2,2.5,3,4,5$, and $6 \mathrm{~h}$ after insulin-loaded DMNRs administration.

\section{Results}

\section{Fabrication and characterization of DMNRs}

The fabrication process of DMNR can be divided into the following three steps (fig. S1, A to G): (1) Drug solution was applied to the PDMS mold and concentrated on the tips of MN cavities under vacuum; (2) $\mathrm{PVA} /$ sucrose solution was cast into the MN cavities under vacuum for $30 \mathrm{~min}$ to fill the drug-loaded cavities of PDMS mold; (3) Drug-loaded MN arrays were demolded using cylindrical rollers with doublesided tape after drying at room temperature. However, it is worth noting that the drying time before demolding is important. In this study, demolding time of DMNRs was optimized and the results are shown in Fig. 1. Obviously, DMNs could not be completely demolded after drying only 2 hours (Fig. 1A), but it could be successfully completely demolded without causing any damage after drying for 4 hours (Fig. 1B) or even 8 hours (Fig. 1C). The relative water content of DMNs demolded at different times was also measured and the result was shown that the relative water content of DMNs demolded at 2 hours was $17.8 \pm 1.2 \%$, and as the drying time increased to 4 hours and 8 hours, the relative water content of DMNs decreased to $9.2 \pm 0.3 \%$ and $6.5 \pm 0.5 \%$, respectively (Fig.1D)

According to the approach described above, five kinds of DMNRs were successfully prepared (Table S1). Microscope images of DMNRs with different heights MNs are illustrated in Fig. 3, and the overview morphology of DMNR with MN height of $600 \mu \mathrm{m}$ was recorded as shown in supporting information (Movie S1). Obviously, the MN geometries remained intact and the tips of MNs were not damaged after demolding via rollers. Besides, by observing the seam morphology of DMNR after being stored for 1 day and 45 days after demolding (fig. S2), it was found that the seam of DMNR after storing 45 days was almost no change compared to the first day after demolding, which is good for DMNR for its long-term storage.

\section{Mechanical performance}

Effective insertion is an important prerequisite for MNs to delivery drug into skin effectively. Several physical factors, such as MN height, density, geometry, have been reported to affect MN mechanical performance (35). In addition, shear force is also an important factor affecting the performance of the 
drug delivery for DMNRs. Hence, the axial force and shear force tests of DMNRs with different heights $(400,600$, and $700 \mu \mathrm{m})$ were performed to characterize the mechanical strength of DMNRs.

The data of the axial force-displacement curves of DMNRs with different height DMNs clearly indicate that the axial force of all the three kinds of DMNRs increased with the increase of displacement, and there was no fracture point for each curve in the range of measurements (Fig. 2E). Microscopic images (Fig. $2 \mathrm{E}$ ) of DMNs after compression further illustrate that there was only a decrease in height of MNs instead of fracturing. Besides, it was worth noting that the axial force of the DMNRs gradually deteriorate as the MN height increased. This decrease mainly due to the higher of the DMN height, the smaller of the DMN diameter near the tip portion, and thus the mechanical property of the DMN is lowered.

Moreover, a shear force was given at the position of $250 \mu \mathrm{m}$ from the tips of the DMNs. As shown in Fig. 3G, the ultimate shear forces for each group of different heights MNs of DMNRs were totally different. For DMNR with MN height of $400 \mu \mathrm{m}$, the MN was fractured when ultimate force reached about $0.90 \mathrm{~N}$; however, for the MNs of $600 \mu \mathrm{m}$ and $700 \mu \mathrm{m}$ height, they were fractured while the average shear force reached about $0.46 \mathrm{~N}$ and $0.20 \mathrm{~N}$, respectively.

\section{Insertion ability of DMNRs in vitro}

To evaluate the in vitro insertion abilities and optimize the height of MNs for efficient drug delivery of DMNRs, porcine cadaver skin without subcutaneous tissues was used as an in vitro skin model and SRBloaded DMNRs with DMN height of 400,600, and $700 \mu \mathrm{m}$ were prepared (Fig. 3, A1 to A3). The porcine cadaver skin surface after administration by DMNRs was imaged in bright field (Fig. 3, B1 to B3) and fluorescence field (Fig. 3, C1 to C3). Obviously, for $400 \mu$ m height DMNs of DMNR, only a small amount of drug was delivered into the skin after rolling the DMNR once across the skin. For $600 \mu \mathrm{m}$ group, the red spots left on the skin were clear and uniform (Fig. 3, B2 and C2). But as the DMN height increased to 700 $\mu \mathrm{m}$, most DMNs were fractured on the skin surface before insertion. (Fig. 3, B3 and C3). Then, the skin sample were sliced from the middle of the DMNRs insertion points and viewed using fluorescence microscope under bright field (Fig. 3, D1 to D3) and fluorescence field (Fig. 3, E1 to E3). These results indicate that the rapid drug delivery ability of DMNR with MN height of $600 \mu \mathrm{m}$ was much better than MNs with height of $400 \mu \mathrm{m}$ or $700 \mu \mathrm{m}$. Therefore, DMNRs with MN height of $600 \mu \mathrm{m}$ were selected for testing in subsequent experiments.

Moreover, to further observe the drug delivery way and holes created of DMNRs with DMN height of 600 $\mu \mathrm{m}$ visually, Parafilm $\mathrm{M}^{\circledR}$ was used as a skin model. After insertion, it could be clearly observed that the drug-loaded tips of DMNs were broke in the Parafilm $M{ }^{\circledR}$ (Movie S2). The insertion holes created of DMNRs could be clearly observed (fig. S3) and it was found that almost all DMNs could penetrate the second layer of Parafilm ${ }^{\circledR}$ and even over $80 \%$ DMNs could penetrate the third layer. Of note, the insertion depth of DMNR on the Parafilm $M^{\circledR}$ is much deeper than on the porcine skin surface. This difference mainly contributed to tips of DMNs were eroded when they touched the interstitial fluid when 
they were inserted into the skin while they were not affected when they were inserted into the Parafilm $M \circledR$.

\section{Rapid drug delivery of DMNR}

Most traditional DMNPs need to be vertically inserted into skin for several minutes or hours to ensure the DMNs complete dissolved $(36,37)$; therefore, the DMNPs are required to be attached to the skin surface all the time during $\mathrm{MN}$ dissolving, which is very inconvenient for patient. In this work, the rapid drug delivery performance of DMNR (MN height is $600 \mu \mathrm{m}$ ) was also assessed. As shown in Fig. 4, compared with the DMNs before insertion (Fig. 4A), only the tips of DMNs produced local surface erosion (Fig. 4, B1 to B3) when the DMNP was vertically inserted into the skin and then removed vertically after 1 second (only suffered from an axial compression force). And the top view (Fig. 4B4) and side view (Fig. 4B5) of the skin after insertion show that only a small amount of model drug was delivered into the skin. Differently, when a roller covered with DMNs of the same geometry to DMNP was rolled across the skin, it can be clearly observed that the drug-loading tips of the MNs were fractured into the skin because of the quick shear force and skin resistance, and there were only a few drug residues on the MNs after insertion (Fig. 4, C1 to C3). Besides, the top view (Fig. 4C4) and side view (Fig. 4C5) of the skin images after insertion by DMNR show that the tips of MNs were then dissolved in the skin and the drug was released into the skin, suggesting that it is unnecessary for DMNR to be attached to the skin surface for several minutes or even longer time like a DMNP and it can deliver the drug into the skin in a rapid manner.

\section{Insertion rate and drug delivery efficiency in vivo}

In addition to shortening drug delivery time, another important goal of ours for DMNR is to expand the application of DMN to enable deliver drugs on the large areas of skin or curved skin. The dorsal skin of SD rats after insertion by the DMNRs containing different numbers of DMNs shows that the number of red spots left on the skin were almost the same as the number of DMNs arranged on DMNRs for each group, indicating the drug delivery performance of DMNRs is less affected as the number of DMNs increased from 225 to 525 for DMNRs. However, for DMNPs, the red spots could be clearly observed on the rat skin surface after insertion by DMNPs with $225 \mathrm{DMNs}$ (Fig. $5 \mathrm{C} 1$ ), but as the number of DMNs increased to 375 , some DMNs near the edge of the DMNP could not be inserted into the rat skin very well (Fig. 5C2), especially when the number of DMNs increased to 525, the number of DMNs near the edge of the DMNP inserted into the skin was significantly reduced due to the skin deformation (Fig. 5C3), suggesting that the drug delivery performance of DMNP was seriously affected by the patch size. Subsequently, the number of red spots created by different arrays of SRB-loaded DMNRs and DMNPs on the insertion sites were separately counted, and the insertion rate was then calculated (Fig. 5D). The results show that although the number of DMNs arranged on the DMNRs was different, the insertion rate on the skin of each group were all over $98 \%$ and there was no significant difference among each group. It shows that although the number of DMNs arranged on the DMNRs was different, the insertion rates on the skin for DMNRs were all over $98 \%$ and almost no significant change among each group; however, for DMN patch, the insertion rate was decreased from $100 \%$ to $93 \%$ as the DMNP enlarged from 225 DMNs 
to 525 DMNs. This difference of insertion rate for the DMNR and the DMNP as mentioned above is mainly due to the following two reasons. First, the drug delivery method of DMNR is rolling the roller across the skin row by row, which is less affected by skin elastic deformation. But for DMNP, all MNs that arranged on the patch need to be vertically inserted into skin at once, which is easy for the small patch but difficult for the large patch because of the skin deformation. Another important reason is that the dorsal skin of rat is arcuate rather than flat so that the roller can be rolled along the curved skin and does not affect the MNs of other rows on the roller, when a small DMNP was inserted into the rat skin, the patch could well fit to the skin (Fig. 5F). In contrast, when a larger patch was applied on the arcuate skin, the MNs arranged on the edge of the patch could not contact well to the skin due to the base of DMNP cannot bend freely (Fig. 5G, marked by the red line circle); therefore, the insertion rate of DMNP on the rat skin was decreased as the $\mathrm{MN}$ patch size enlarged.

\section{Transdermal drug delivery}

The fluorescence intensity of model drug delivered into the dorsal skin of SD rat using DMNR and DMNP were visualized by IVIS. The fluorescent intensity of both DMNP (left) and DMNR (right) treated skin decreased over time (Fig. 5l), indicating that the model drug gradually diffused into the deep tissues of skin. Remarkably, the skin fluorescence intensity at $0 \mathrm{~h}$ after DMNR treatment was observed to be more uniform than that of DMNP treatment because the MNs arranged on the roller were still in good contact with curved skin, while the MNs arranged at the edge of the patch did not.

\section{Hypoglycemic effect of insulin-loaded DMNRs for diabetic rats}

To assess the practical application of the DMNRs, the insulin-loaded DMNR was applied to deliver insulin to the STZ-induced SD diabetic rats. The blood glucose level changes of diabetic rats can reflect whether the drug was successfully delivered into the skin indirectly. The curves of time versus blood glucose level (\% of initial level) after subcutaneous (SC) injection and DMNRs administration of insulin are shown in Fig. 6 . The blood glucose level of control group was almost no change after transdermal administration of insulin-unloaded DMNRs to the diabetic rats (control). In contrast, for insulin-loaded DMNRs administration group (insulin DMNRs, $0.3 \mathrm{IU}$ ), the blood glucose level was dramatically reduced blow $50 \%$ at $1 \mathrm{~h}$ after insulin-loaded DMNR administrated and reached minimum level at $1.5 \mathrm{~h}$, then return to initial level after $6 \mathrm{~h}$. The hypoglycemic effect tendency of insulin-loaded DMNRs was similar to the SC injection group's (SC injection, $0.3 \mathrm{IU}$ ). It demonstrates that the insulin-loaded DMNR displayed a good potential for rapid insulin delivery to diabetes.

\section{Discussion}

To enable DMNs for drug delivery in a rapid and highly efficient manner on the skin, especially on the large areas of skin or curved skin, a DMNR device was designed, which is not required to be attached to the skin surface during drug delivery and can be used for rapid and highly efficient drug delivery because of its high mobility and ability to cover large surface areas. Compared to the traditional DMNPs preparation process (fig. S1), the fabrication process of DMNRs was only changed the demolding method 
using a roller with double-side tape instead of a small patch to arrange the DMNs, which is easy to operate, no heating required and low-cost.

Demolding time of DMNRs was researched firstly and it was found that the DMNs could be successfully demolded from PDMS molds after drying 4 hours or even 8 hours. Then, an optimized DMN height was obtained according to the mechanical performance test and in vivo skin insertion test. The $600 \mu \mathrm{m}$ height DMN shows excellent skin insertion ability and drug delivery performance. Parafilm $\mathrm{M}^{\circledR}$ after insertion by DMNR (Movie S2) and the DMNs morphology of the DMNR after it was rolled once across the pig cadaver skin (Fig. 4) then confirmed that the main way for DMNR to deliver drugs is mainly due to fracture of DMNs under a shear force and skin resistance, and the drug-loaded tips was first fractured into the skin and then dissolved to release drugs, which is convenient and no risk of infection or erythema caused by the patch being attached to the skin for a long time, especially for the skin sensitive patients.

Next, the insulin-loaded DMNRs were used for type 1 diabetic rats treatment. The hypoglycemic effect tendency of insulin-loaded DMNRs was similar to the SC group's. These results indicated that the DMNR, as a safe and rapid drug delivery device, could provide a solution to the problems of drug delivery on the large skin or curved skin. For future, it is expected to be used for delivering drug for diabetes, some large skin diseases such as psoriasis or other purposes such as cosmetic.

\section{Declarations}

\section{Ethics approval and consent to participate}

The experimental protocol was established, according to the ethical guidelines of the BUCT Declaration and was approved by the Ethics Committee of Beijing University of Chemical Technology. Written informed consent was obtained from individual or guardian participants.

\section{Consent for publication}

Not applicable.

\section{Availability of data and materials}

All data generated or analysed during this study are included in this work.

\section{Authors' contributions}

Xiao Peng Zhang: Writing- Original draft preparation. Bao Li Zhang: Methodology. Bo Zhi Chen: Data curation. Ze Qiang Zhao: Data Curation. Xin Dong Guo: Conceptualization, Project administration.

\section{Acknowledgements}

Funding: This work was financially supported by the National Natural Science Foundation of China (51873015), and the long-term subsidy mechanism from the Ministry of Finance and the Ministry of 
Education of PRC.

Competing interests: The authors declare that they have no competing interests.

\section{References}

1. M. R. Prausnitz, S. Mitragotri, R. Langer, Current status and future potential of transdermal drug delivery. Nat. Rev. Drug Discov. 3, 115-124 (2004)

2. M.-H. Ling, M.-C. Chen, Dissolving polymer microneedle patches for rapid and efficient transdermal delivery of insulin to diabetic rats. Acta Biomater. 9, 8952-8961 (2013)

3. M. R. Prausnitz, R. Langer, Transdermal drug delivery. Nat. Biotechnol. 26, 1261-1268 (2008)

4. T. Hsu, S. Mitragotri, Delivery of siRNA and other macromolecules into skin and cells using a peptide enhancer. Proc. Natl. Acad. Sci. U. S. A. 108, 15816-15821 (2011)

5. H. L. Quinn, M. C. Kearney, A. J. Courtenay, M. T. C. McCrudden, R. F. Donnelly, The role of microneedles for drug and vaccine delivery. Expert Opin. Drug Deliv. 11, 1769-1780 (2014)

6. S. Bhatnagar, K. Dave, V. V. K. Venuganti, Microneedles in the clinic. J. Control. Release 260, 164-182 (2017)

7. Y.-C. Kim, J.-H. Park, M. R. Prausnitz, Microneedles for drug and vaccine delivery. Adv. Drug Deliv. Rev. 64, 1547-1568 (2012)

8. K. Lee, C. Y. Lee, H. Jung, Dissolving microneedles for transdermal drug administration prepared by stepwise controlled drawing of maltose. Biomaterials 32, 3134-3140 (2011)

9. T. Waghule, G. Singhvi, S. K. Dubey, M. M. Pandey, G. Gupta, M. Singh, K. Dua, Microneedles: A smart approach and increasing potential for transdermal drug delivery system. Biomed. Pharmacother. 109, 1249-1258 (2019)

10. S. Dharadhar, A. Majumdar, S. Dhoble, V. Patravale, Microneedles for transdermal drug delivery: a systematic review. Drug Dev. Ind. Pharm. 45, 188-201 (2019)

11. Y. Ye, J. Yu, D. Wen, A. R. Kahkoska, Z. Gu, Polymeric microneedles for transdermal protein delivery. Adv. Drug Deliv. Rev. 127, 106-118 (2018)

12. Q. Y. Li, J. N. Zhang, B. Z. Chen, Q. L. Wang, X. D. Guo, A solid polymer microneedle patch pretreatment enhances the permeation of drug molecules into the skin. RSC Advances 7, 1540815415 (2017)

13. Y. Chen, B. Z. Chen, Q. L. Wang, X. Jin, X. D. Guo, Fabrication of coated polymer microneedles for transdermal drug delivery. J. Control. Release 265, 14-21 (2017)

14. A. M. Rodgers, A. J. Courtenay, R. F. Donnelly, Dissolving microneedles for intradermal vaccination: manufacture, formulation, and stakeholder considerations. Expert Opin. Drug Deliv. 15, 1039-1043 (2018)

15. H. Chang, M. J. Zheng, X. J. Yu, A. Than, R. Z. Seeni, R. J. Kang, J. Q. Tian, D. P. Khanh, L. B. Liu, P. Chen, C. J. Xu, A swellable microneedle patch to rapidly extract skin interstitial fluid for timely 
metabolic analysis. Adv. Mater. 29, 8 (2017)

16. L. Niu, L. Y. Chu, S. A. Burton, K. J. Hansen, J. Panyam, Intradermal delivery of vaccine nanoparticles using hollow microneedle array generates enhanced and balanced immune response. J. Control. Release 294, 268-278 (2019)

17. Q. Y. Yan, Z. G. Chen, H. M. Liu, W. S. Shan, Z. D. Cheng, X. Y. Dai, Y. Xue, F. Chen, Enhancement of Ag85B DNA vaccine immunogenicity against tuberculosis by dissolving microneedles in mice. Vaccine 36, 4471-4476 (2018)

18. G. Kang, S. Kim, H. Yang, M. Jang, L. Chiang, J. H. Baek, J. H. Ryu, G. W. Choi, H. Jung, Combinatorial application of dissolving microneedle patch and cream for improvement of skin wrinkles, dermal density, elasticity, and hydration. J. Cosmet. Dermatol. 18, 1083-1091 (2019)

19. C. Dillon, H. Hughes, N. J. O'Reilly, C. J. Allender, D. A. Barrow, P. McLoughlin, Dissolving microneedle based transdermal delivery of therapeutic peptide analogues. Int. J. Pharm. 565, 9-19 (2019)

20. J. Y. Kim, M. R. Han, Y. H. Kim, S. W. Shin, S. Y. Nam, J. H. Park, Tip-loaded dissolving microneedles for transdermal delivery of donepezil hydrochloride for treatment of Alzheimer's disease. Eur. J. Pharm. Biopharm. 105, 148-155 (2016)

21. D. Wu, H. Katsumi, Y. S. Quan, F. Kamiyama, K. Kusamori, T. Sakane, A. Yamamoto, Permeation of sumatriptan succinate across human skin using multiple types of self-dissolving microneedle arrays fabricated from sodium hyaluronate. J. Drug Target. 24, 752-758 (2016)

22. B. Z. Chen, M. Ashfaq, X. P. Zhang, J. N. Zhang, X. D. Guo, In vitro and in vivo assessment of polymer microneedles for controlled transdermal drug delivery. J Drug Target 26, 720-729 (2018)

23. E. Jacoby, C. Jarrahian, H. F. Hull, D. Zehrung, Opportunities and challenges in delivering influenza vaccine by microneedle patch. Vaccine $33,4699-4704$ (2015)

24. M. T. C. Mc Crudden, E. Larraneta, A. Clark, C. Jarrahian, A. Rein-Weston, S. Lachau-Durand, N. Niemeijer, P. Williams, C. Haeck, H. O. McCarthy, D. Zehrung, R. F. Donnelly, Design, formulation and evaluation of novel dissolving microarray patches containing a long-acting rilpivirine nanosuspension. J. Control. Release 292, 119-129 (2018)

25. A. Abramson, E. Caffarel-Salvador, V. Soares, D. Minahan, R. Y. Tian, X. Y. Lu, D. Dellal, Y. Gao, S. Kim, J. Wainer, J. Collins, S. Tamang, A. Hayward, T. Yoshitake, H.-C. Lee, J. Fujimoto, M. R. Frederiksen, U. Rahbek, N. Roxhed, R. Langer, G. Traverso, A luminal unfolding microneedle injector for oral delivery of macromolecules. Nat. Med. 25, 1512-+ (2019)

26. H. Kathuria, H. L. Li, J. P. Pan, S. H. Lim, J. S. Kochhar, C. Wu, L. F. Kang, Large size microneedle patch to deliver lidocaine through skin. Pharm. Res. 33, 2653-2667 (2016)

27. H. Miki, R. Nishikata, K. Minehira, S. Tsuchitani, Novel structure of microneedle arrays for the transdermal drug delivery applications. IEEJ Trans. Electr. Electron. Eng. 14, 163-164 (2019)

28. J. Yoon, T. Son, E.-h. Choi, B. Choi, J. S. Nelson, B. Jung, Enhancement of optical skin clearing efficacy using a microneedle roller. J. Biomed. Opt. 13, 021103 (2008)

29. D. Huang, D. Y. Zhao, X. X. Wang, C. H. Li, T. R. Yang, L. L. Du, Z. W. Wei, Q. Cheng, H. Q. Cao, Z. C. Liang, Y. Y. Huang, Z. H. Li, Efficient delivery of nucleic acid molecules into skin by combined use of 
microneedle roller and flexible interdigitated electroporation array. Theranostics 8, 2361-2376 (2018)

30. J.-H. Park, S.-O. Choi, S. Seo, Y. B. Choy, M. R. Prausnitz, A microneedle roller for transdermal drug delivery. Eur. J. Pharm. Biopharm. 76, 282-289 (2010)

31. M. C. Aust, K. Reimers, H. M. Kaplan, F. Stahl, C. Repenning, T. Scheper, S. Jahn, N. Schwaiger, R. Ipaktchi, J. Redeker, M. A. Altintas, P. M. Vogt, Percutaneous collagen induction-regeneration in place of cicatrisation? J. Plast. Reconstr. Aes. 64, 97-107 (2011)

32. T. S. Alster, P. M. Graham, Microneedling: A review and practical guide. Dermatol. Surg. 44, 397-404 (2018)

33. J. Nguyen, K. B. Ita, M. J. Morra, I. E. Popova, The influence of solid microneedles on the transdermal delivery of selected antiepileptic drugs. Pharmaceutics 8, UNSP 33 (2016)

34. A. Omolu, M. Bailly, R. M. Day, Assessment of solid microneedle rollers to enhance transmembrane delivery of doxycycline and inhibition of MMP activity. Drug Deliv. 24, 942-951 (2017)

35. M.-C. Chen, M.-H. Ling, K.-Y. Lai, E. Pramudityo, Chitosan microneedle patches for sustained transdermal delivery of macromolecules. Biomacromolecules 13, 4022-4031 (2012)

36. D. D. Zhu, Q. L. Wang, X. B. Liu, X. D. Guo, Rapidly separating microneedles for transdermal drug delivery. Acta Biomater. 41, 312-319 (2016)

37. P. González-Vázquez, E. Larrañeta, M. T. C. McCrudden, C. Jarrahian, A. Rein-Weston, M. QuintanarSolares, D. Zehrung, H. McCarthy, A. J. Courtenay, R. F. Donnelly, Transdermal delivery of gentamicin using dissolving microneedle arrays for potential treatment of neonatal sepsis. J. Control. Release 265, 30-40 (2017)

38. Q. L. Wang, D. D. Zhu, Y. Chen, X. D. Guo, A fabrication method of microneedle molds with controlled microstructures. Mater. Sci. Eng. C 65, 135-142 (2016)

39. E. Larrañeta, J. Moore, E. M. Vicente-Perez, P. Gonzalez-Vazquez, R. Lutton, A. D. Woolfson, R. F. Donnelly, A proposed model membrane and test method for microneedle insertion studies. Int. J. Pharm. 472, 65-73 (2014)

\section{Figures}



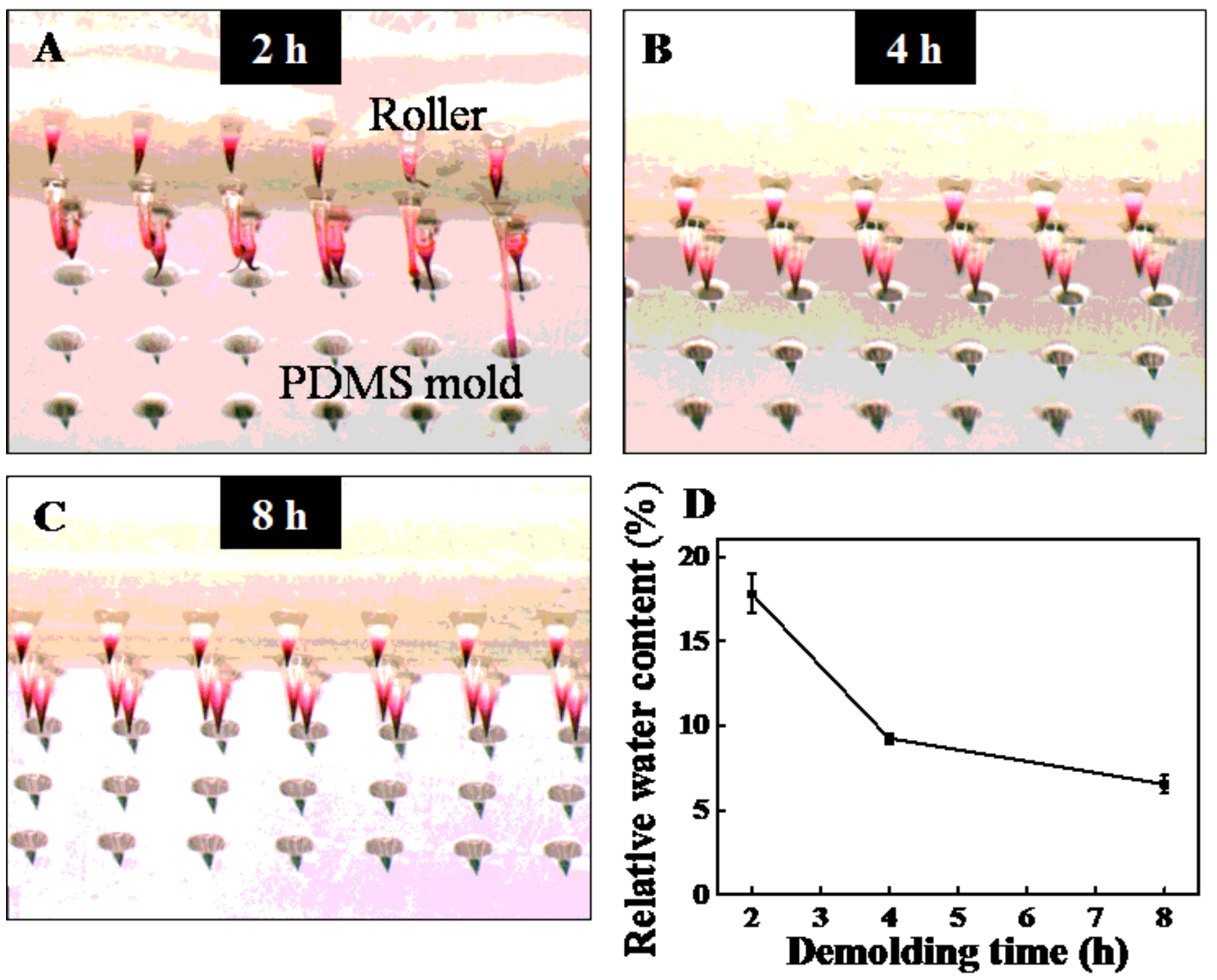

Figure 1

Demolding time study of DMNR. Demolding of DMNR from PDMS mold after drying $2 \mathrm{~h}(\mathrm{~A}), 4 \mathrm{~h}(\mathrm{~B})$, and 8 $h(C)$. (D)The corresponding relative water content (\%) of MNs after drying different times before demolding. 

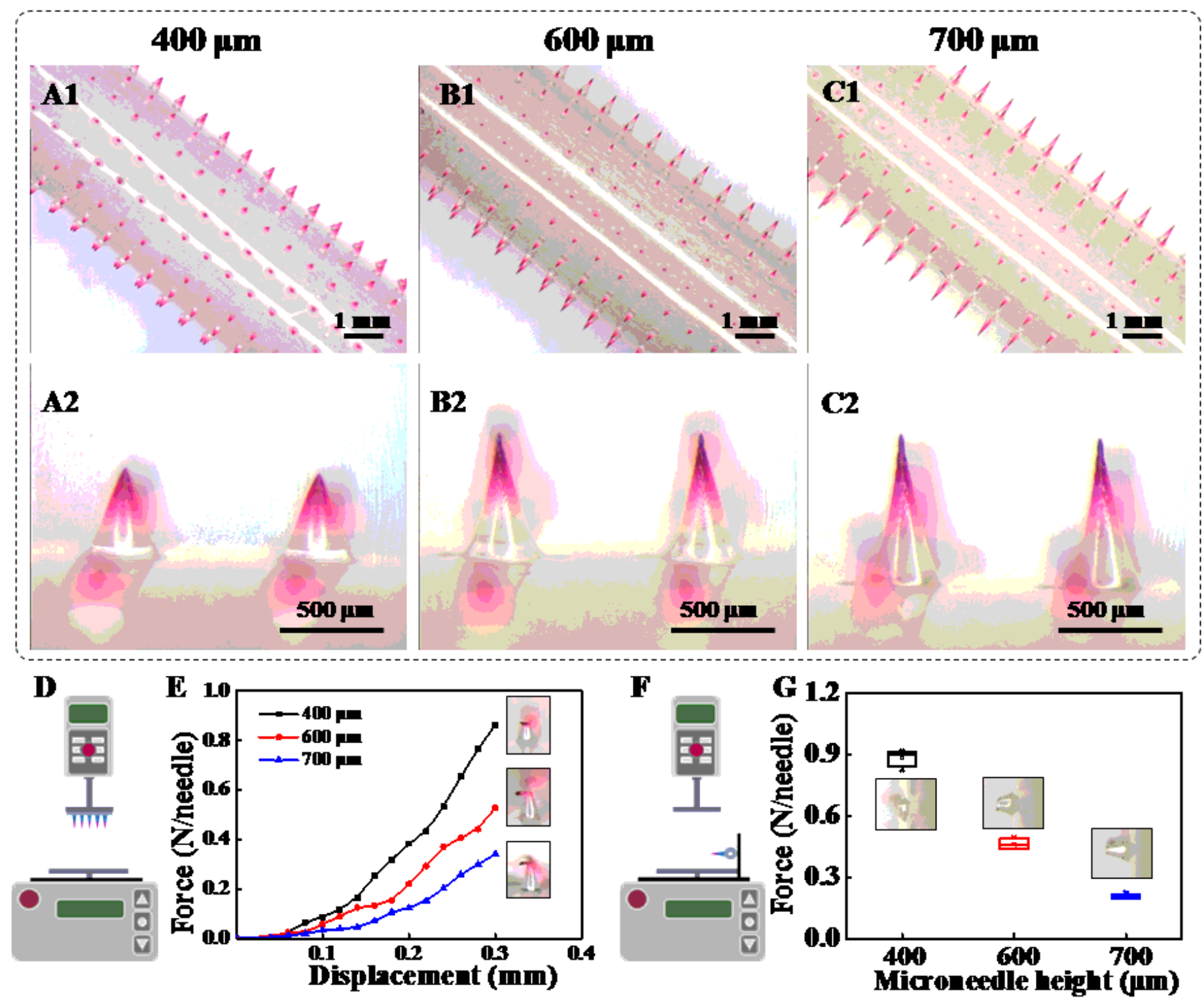

Figure 2

Microscope images and mechanical performance of DMNRs with different heights MNs. (A1, A2) DMNR with MN height of $400 \mu \mathrm{m}$. (B1, B2) DMNR with MN height of $600 \mu \mathrm{m}$. (C1, C2) DMNR with MN height of $700 \mu \mathrm{m}$. (D)Schematic diagrams of axial force tests of MNs. (E) The force-displacement curves of DMNRs with different height of MNs under axial force. (F) Schematic diagrams of shear force tests of MNs. (G) The ultimate force data when the MNs were fractured under the shear force. 


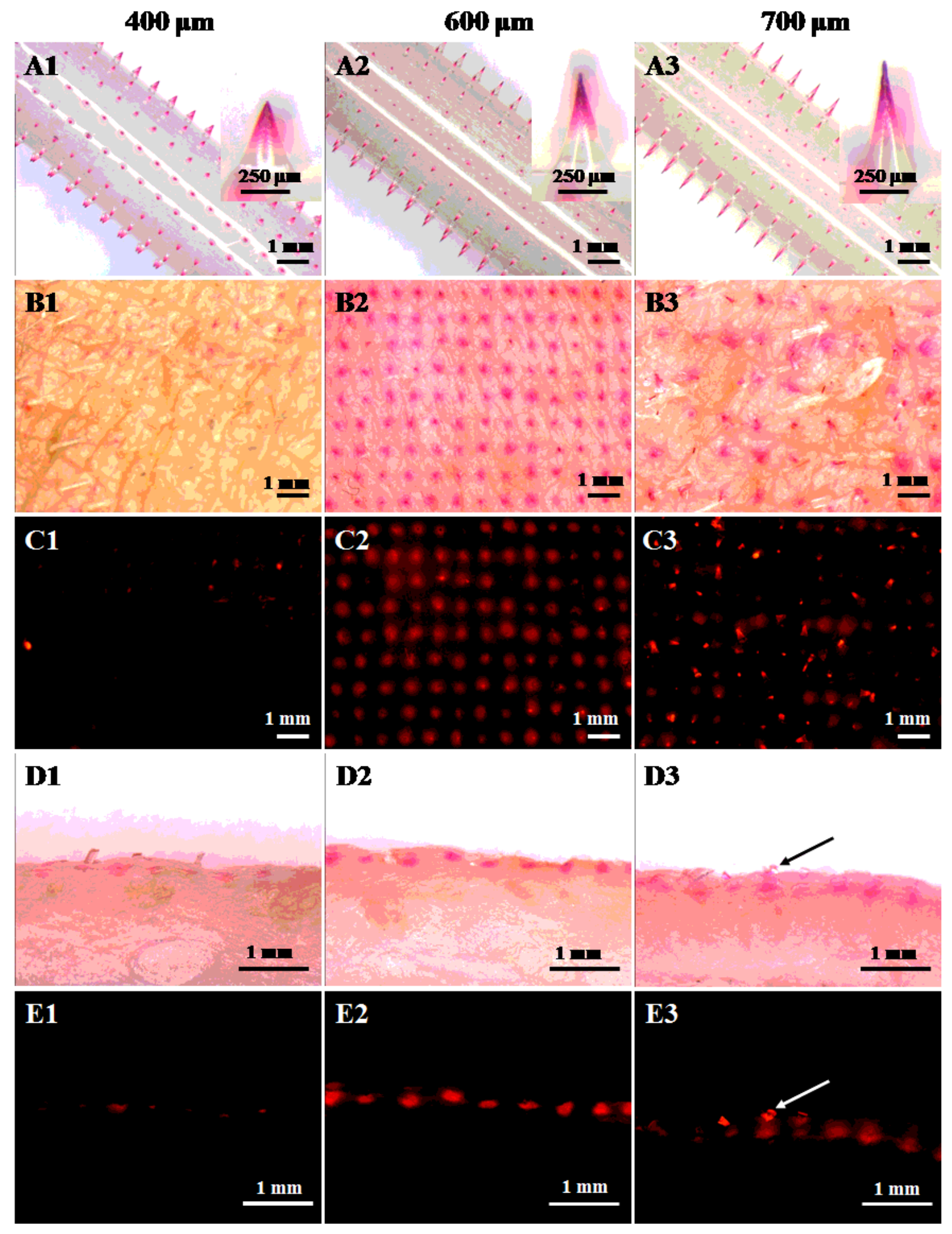

Figure 3

Microscope pictures of DMNRs insertion tests in vitro. (A1-A3) SRB-loaded DMNR with MN heights of 400, 600 and $700 \mu \mathrm{m}$, respectively. The bright field (B1-B3) and fluorescence field (C1-C3) images of the porcine cadaver skin after insertion by DMNRs with different height MNs (400,600 and $700 \mathrm{um})$, and the corresponding side views of the insertion sites in the skin were imaged in bright field (D1- D3) and fluorescence field (E1-E3). 


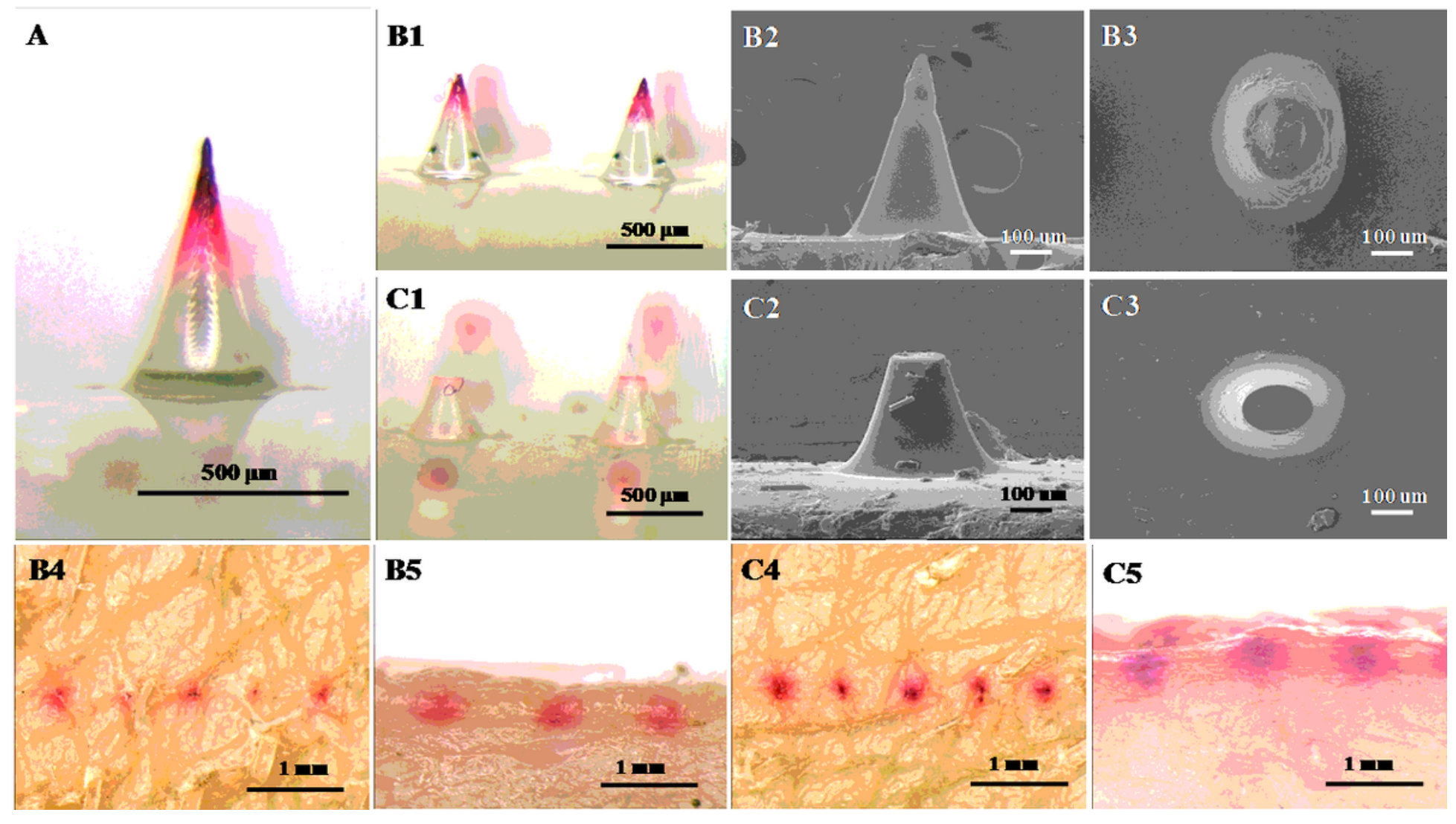

Figure 4

Rapid drug delivery of DMNR. (A) The DMN image before insertion. (B1) Image of DMNs after DMNP vertically inserted into the pig cadaver skin and then removed vertically at 1 second (DMNP group). (B2, B3) The side view and top view SEM images of DMNs after DMNP vertically inserted into the skin and then removed vertically at 1 second. (B4) Top view and (B5) side view of the skin after administrated 1 second by DMNP. (C1) The images of DMNs after DMNR inserted the skin by rolling it once across the pig cadaver skin and removed within 1 second. (C2, C3) The side view and top view SEM images of DMNs after DMNR inserted the skin by rolling it once across the pig cadaver skin and removed within 1 second. The top view (C4) and side view (C5) images of the skin after administrated by DNMR. 

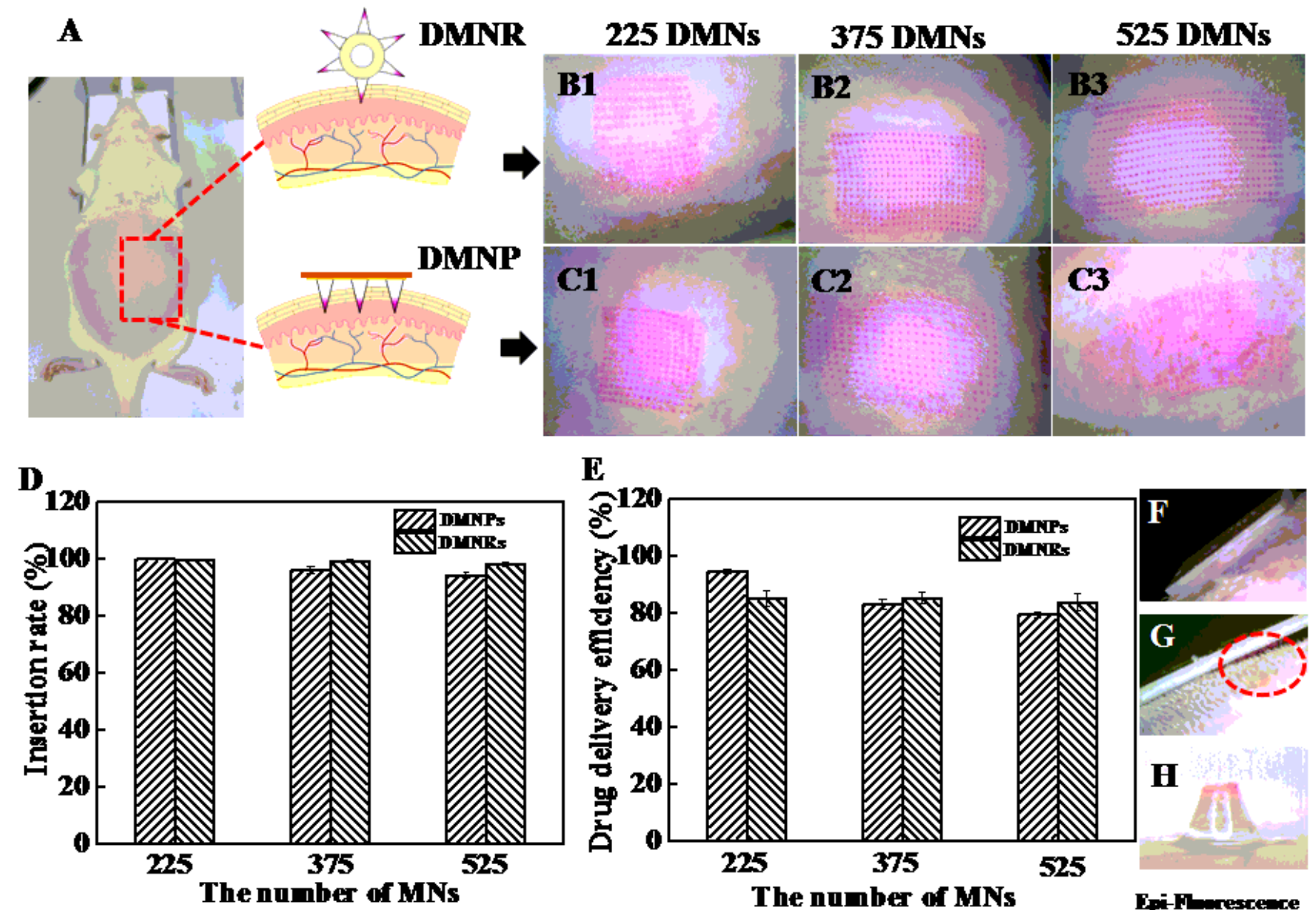

$\mathbf{E}$

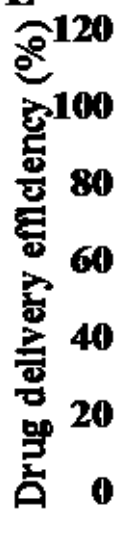

$\mathbf{I}$
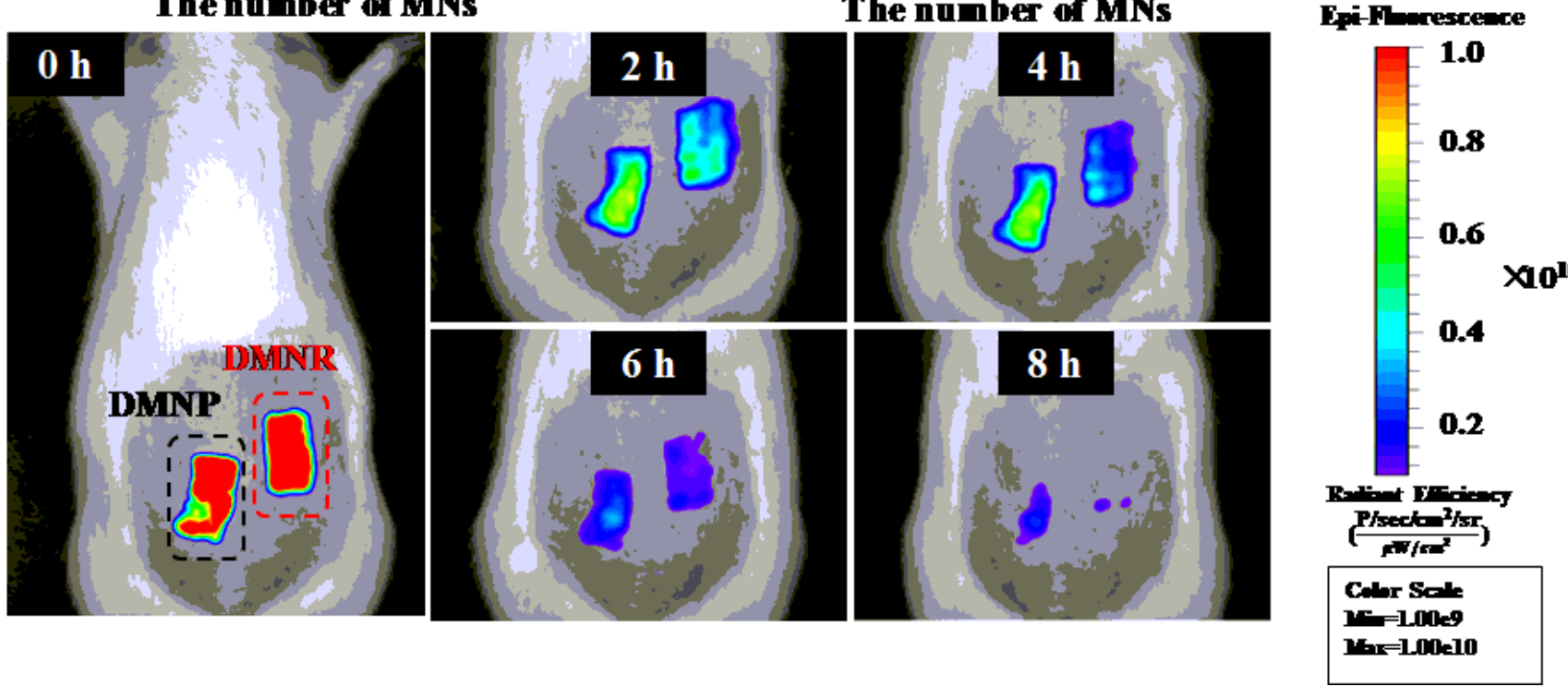

Figure 5

Insertion ability and drug delivery performance of DMNRs and DMNP in vivo. (A) The schematic diagram of the drug delivery sites using DMNRs and DMNPs. (B1-B3) The dorsal skin pictures of SD rats after insertion by DMNRs with 225 DMNs (B1), 375 DMNs (B2), and 525 DMNs (B3). (C1-C3) The dorsal skin pictures of SD rats skin after insertion by DMNPs with 225 DMNs (C1), 375 DMNs (C2), and 525 DMNs (C3). (D) Insertion rate of DMNRs and DMNPs. (E) Drug delivery efficiency of DMNRs and DMNPs. (F) $15 \times$ 15 array of DMNP applied to the dorsal skin of SD rat. (G) $15 \times 35$ arrays DMNP applied to the dorsal skin 
of SD rat. (H) DMN morphology of DMNRs after rolled across the dorsal skin of rat. (I) In vivo fluorescence images of SD rats at different time $(0,2,4,6$ and $8 \mathrm{~h})$ after treatment by DMNP (left) and DMNR (right). The MN heights of all groups are $600 \mu \mathrm{m}$. Data are presented as the average values \pm standard deviation.

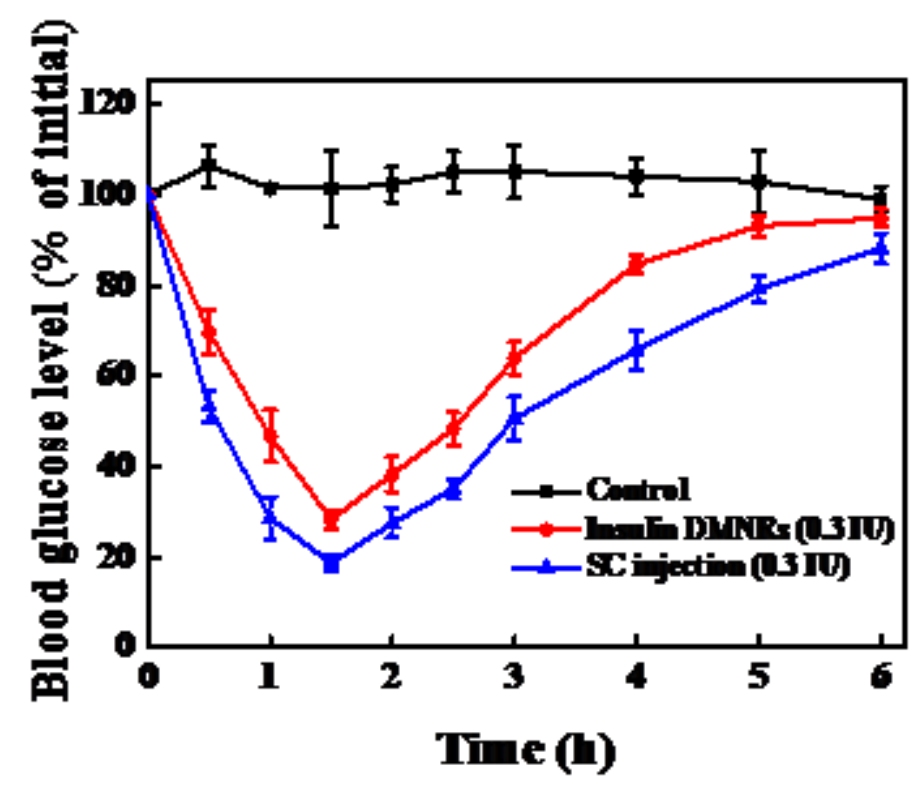

Figure 6

In vivo evaluation of insulin-loaded DMNR for type 1 diabetic rats treatment. Blood glucose levels versus time profiles of diabetic rats after administration of insulin-unloaded DMNRs (control), 0.3 IU insulinloaded DMNRs (Insulin DMNRs, 0.3 IU), and subcutaneous injection (SC injection,0.3IU). Data are presented as the average values \pm standard deviation ( $n=4$ for each group).

\section{Supplementary Files}

This is a list of supplementary files associated with this preprint. Click to download.

- GA.png

- MovieS1.mp4

- MovieS2.mp4

- supportinginformation.docx 